\title{
Distributed Consensus in Networks of Dynamic Agents
}

\author{
D. Bauso, L. Giarré and R. Pesenti
}

\begin{abstract}
Stationary and distributed consensus protocols for a network of $n$ dynamic agents under local information is considered. Consensus must be reached on a group decision value returned by a function of the agents' initial state values. As a main contribution we show that the agents can reach consensus if the value of such a function computed over the agents' state trajectories is time invariant. We use this basic result to introduce a protocol design rule allowing consensus on a quite general set of values. Such a set includes, e.g., any generalized mean of order $p$ of the agents' initial states. We demonstrate that the asymptotical consensus is reached via a Lyapunov approach. Finally we perform a simulation study concerning the alignment maneuver of a team of unmanned air vehicles.
\end{abstract}

\section{INTRODUCTION}

Distributed consensus protocols are local control policies based on partial information that allow the coordination of multi-agent systems. Agents implement a consensus protocol to reach consensus, that is to (make their states) converge to a same value, called consensus-value, or group decision value [1].

Coordination of agents/vehicles is an important task in several applications including autonomous formation flight [2], [3], cooperative search of unmanned air-vehicles (UAVs) [4], [5], swarms of autonomous vehicles or robots [6], [7], multi-retailer inventory control [8], [9] and congestion/flow control in communication networks [10].

Actually, a central point in consensus problems is the connection between the graph structure, defined by the Laplacian Matrix, and delays or distortions in communication links [11]. Switching topology and directional communications are studied in [1], [12], [13], [14], [15], [16], while cooperation based on the notion of coordination variable and coordination function in [17], [18]. There, coordination variable is referred to as the minimal amount of information needed to effect a specific coordination objective, whereas a coordination function parameterizes the effect of the coordination variable on the myopic objectives of each agent.

In this paper, $n$ dynamic agents reach consensus on a group decision value by implementing distributed and stationary control policies based on neighbors' state feedback. Here, neighborhood relations are defined by a time-invariant connected undirected communication topology. To generalize our results for switching topology and directional communications, one could follow the

This work was supported by MIUR-PRIN "Robust Techniques for uncertain systems control"

D. Bauso and R. Pesenti are with DINFO, Università di Palermo, Italy pesenti@unipa.it

L. Giarré is with DIAS, Università di Palermo, Italy giarre@unipa.it same line of reasoning as in [1]. Similarly to [1], [2], [19], the dynamics of the agents is a simple first order one. We restrict the group decision value to be a permutation invariant function of the agents' state initial values. Permutation invariance means that the value of the function is independent of the agents indexes.

Our contribution to the study on consensus problems is to show that consensus can be reached if the agents' state trajectories satisfy a certain time invariancy property. On the basis of such a result, we prove that the group decision values considered are sufficiently general to include any mean of order $p$ of the agents' state initial values, such as the arithmetic/min/max means usually dealt with in the literature (see, e.g., [20]). Finally we argue that agents reach asymptotically consensus on the desired group decision value by studying equilibrium properties and stability of the group decision value via Lyapunov theory.

\section{The Consensus Problem}

We consider a system of $n$ agents $\Gamma=\{1, \ldots, n\}$ and model the interaction topology among agents through a time-invariant connected undirected graph $G=(\Gamma, E)$. The graph is undirected since we assume the existence of only bidirectional information exchange links between pairs of agents. Then, each edge $(i, j)$ in the edgeset $E$ means that agent $i$ can receive information from agent $j$ and, vice versa, agent $j$ can receive information from agent $i$. The graph is connected since we assume that for any agent $i \in \Gamma$ there exists a path, i.e., a sequence of edges in $E,\left(i, k_{1}\right)\left(k_{1}, k_{2}\right) \ldots\left(k_{r}, j\right)$, that connects it with any other agent $j \in \Gamma$. Finally, the graph $G$ is not complete since each agent $i$ exchanges information only with a subset of other agents $N_{i}=\{j:(i, j) \in E\}$ called neighborhood of $i$.

Each agent $i$ has a (simplified) first-order dynamics controlled by a distributed and stationary control policy

$$
\dot{x}_{i}=u_{i}\left(x_{i}, x^{(i)}\right) \quad \forall i \in \Gamma,
$$

where $x^{(i)}$ is the state vector of the agents in $N_{i}$ with generic component $j$ defined as follows, $x_{j}^{(i)}=$ $\begin{cases}x_{j} & \text { if } j \in N_{i} \\ 0 & \text { otherwise }\end{cases}$

The policy is distributed since, for each agent $i$, it depends only on the local information available to it, which is $x_{i}$ and $x^{(i)}$. No other information on the current or past system state is available to agent $i$. (We discuss the limit of this assumption at the beginning of Section 3). The policy is stationary since it does not depend explicitly on time $t$. In other words, the policy is a time invariant 
and memoryless function of the state. Define the vector $x(t)=\left\{x_{i}(t), i \in \Gamma\right\}$ as the system state and $u()=$. $\left\{u_{i}():. i \in \Gamma\right\}$ as a distributed stationary protocol or simply a protocol. In accordance with [1], we say that a protocol $u($.$) makes the agents asymptotically reach$ consensus on a group decision value $\hat{\chi}(x(0))$ if there exists an asymptotically stable equilibrium $x^{*}$ of $\dot{x}=u(x)$, such that $x^{*}=\hat{\chi}(x(0)) \mathbf{1}$. When this happens we also say that the system converges to $\hat{\chi}(x(0)) \mathbf{1}$. Here and in the following, 1 stands for the vector $(1,1, \ldots, 1)^{T}$.

Notwithstanding each agent $i$ has only a local information $\left(x_{i}, x^{(i)}\right)$ on the system state $x$, we are interested in making the agents reach consensus on group decision values that are functions of the whole system initial state $x(0)$. In particular, we study protocols that make the agents asymptotically reach consensus on a group decision value $\hat{\chi}(x(0))$ where $\hat{\chi}: \Re^{n} \rightarrow \Re$ is a generic function, such that

$$
\min _{i \in \Gamma}\left\{y_{i}\right\} \leq \hat{\chi}(y) \leq \max _{i \in \Gamma}\left\{y_{i}\right\}, \quad \text { for all } y \in \Re^{n} .
$$

In other words, the group decision value must be confined between the minimum and the maximum agents' initial state values. Any values in this range can be chosen as consensus value by the general protocol we consider. Henceforth the function $\hat{\chi}($.$) will be also referred to as agreement function.$ In the above context, we face the following problem.

Problem 1: (Consensus Problem) Consider a network $G=(\Gamma, E)$ of dynamic agents with first-order dynamics. For any function $\hat{\chi}($.$) determine a (distributed stationary)$ protocol, whose components have the feedback form (1), that makes the agents asymptotically reach consensus on $\hat{\chi}(x(0))$ for any initial condition $x(0)$.

In the following, a protocol that solves the consensus problem is also referred to as a consensus protocol.

\section{TIME INVARIANCY OF $\hat{\chi}(x(t))$}

Initially, we show that if a protocol, which solves a consensus problem, is distributed and stationary then the system state trajectory enjoys the property that $\hat{\chi}(x(t))$ is time invariant. Then, we find a family of non trivial protocols that guarantee such a property. We prove that some of such protocols are consensus protocols with respect to $\hat{\chi}(x(0))$ in the next section.

Lemma 1: (Time invariancy) Consider a network $G=$ $(\Gamma, E)$ of dynamic agents with first-order dynamics. For any function $\hat{\chi}($.$) implement a distributed stationary protocol$ $u(t)$, whose components have the feedback form (1), that makes the agents converge to value $\hat{\chi}(x(0))$ for any initial state $x(0)$. Then the value of $\hat{\chi}(x(t))$ is time invariant, i.e., $\hat{\chi}(x(t))=\hat{\chi}(x(0))$ for all $t>0$.

Observe that there may exist consensus protocols not implying the time invariancy of $\hat{\chi}(x(t))$. However, such protocols must rely on additional information about the whole system initial state $x(0)$ or the value of $\hat{\chi}(x(0))$ (see, e.g., [21] where a local estimate of the percentage of the active players at each stage is performed). Unfortunately,

\begin{tabular}{|lccc|}
\hline mean & $\hat{\chi}(x)$ & $f(y)$ & $g(z)$ \\
\hline arithmetic & $\sum_{i \in \Gamma} \frac{1}{n} x_{i}$ & $\frac{1}{n} y$ & $z$ \\
geometric & $\sqrt[n]{\prod_{i \in \Gamma} x_{i}}$ & $e^{\frac{1}{n} y}$ & $\log z$ \\
harmonic & $\sum_{i \in \Gamma} \frac{n}{x_{i}}$ & $\frac{n}{y}$ & $\frac{1}{z}$ \\
mean of order $p$ & $\sqrt[p]{\sum_{i \in \Gamma} \frac{1}{n} x_{i}^{p}}$ & $\sqrt[q]{\frac{1}{n} y}$ & $z^{p}$ \\
\hline
\end{tabular}

TABLE I

COMMONLY USED MEANS AND THEIR REPRESENTATIONS IN TERMS OF $f$ AND $g$

in the case under study the local information alone is in general not sufficient to reconstruct either $x(0)$ or $\hat{\chi}(x(0))$.

Supposing that the state trajectory reaches the point $\hat{\chi}(x(0)) \mathbf{1}$, the time invariancy property stated in Lemma 1 implies also that $\hat{\chi}(x(0))=\hat{\chi}(\hat{\chi}(x(0)) \mathbf{1})$. Note that the last condition satisfies (2). Actually, (2) imposes that function $\hat{\chi}($.$) must be chosen such that any point \lambda \mathbf{1}$, for all $\lambda \in \Re$, is a fixed point, i.e., $\hat{\chi}(\lambda \mathbf{1})=\lambda$, as it can be trivially derived assuming $x(0)=\lambda \mathbf{1}$.

With this consideration in mind, let us impose the time invariancy of $\hat{\chi}(x(t))$. It holds $\hat{\chi}(x(t))=$ const when

$\frac{d \hat{\chi}(x(t))}{d t}=\nabla_{x} \hat{\chi}(x) \cdot \dot{x}=\sum_{i \in \Gamma} \frac{\partial \hat{\chi}(x)}{\partial x_{i}} \dot{x}_{i}=\sum_{i \in \Gamma} \frac{\partial \hat{\chi}(x)}{\partial x_{i}} u_{i}=0$.

The trivial protocol constantly equal to 0 leaves any value $\hat{\chi}(x(t))$ time invariant, for any possible $\hat{\chi}($.$) , but obviously$ does not make the system converge. Consequently, it is no longer considered hereafter.

Some other solutions of equation (3) can be obtained easily when $\hat{\chi}(x)$ presents a particular structure. A first possibility is when the following condition holds

$$
\frac{\partial \hat{\chi}(x)}{\partial x_{i}} u_{i}=0 \quad \forall i \in \Gamma \text {. }
$$

For example, $\hat{\chi}(x)=\min \left\{x_{i}\right\}$ and $u_{i}=$ $h\left(x_{i}, \min _{j \in N_{i}}\left\{x_{j}\right\}\right)$ satisfy the above condition, for any $h(x, y): \Re^{2} \rightarrow \Re$ such that $h(x, y)=0$ when $x=y$. Actually, $\frac{\partial \hat{\chi}(x)}{\partial x_{i}} \neq 0$ only for $i$ such that $x_{i}=\min _{j \in \Gamma}\left\{x_{j}\right\}$, then, by definition of function $h($.$) , it holds u_{i}\left(x_{i}\right)=0$ and hence (4). The system converges to $\hat{\chi}(x(0))$ if we impose the additional condition $h(x, y)<0$ when $x>y$. Trivially, analogous argument applies to $\hat{\chi}(x)=\max \left\{x_{i}\right\}$. We specialize our study considering a more general family of function $\hat{\chi}(x)$.

Assumption 1: (Structure of $\hat{\chi}($.$) ) Assume \hat{\chi}($.$) satisfy-$ ing condition (2) and such that $\hat{\chi}(x)=f\left(\sum_{i \in \Gamma} g\left(x_{i}\right)\right)$, for some $f, g: \Re \rightarrow \Re$ with $\frac{d g\left(x_{i}\right)}{d x_{i}} \neq 0$ for all $x_{i}$.

This set of functions includes all the commonly used means, at least when $x_{i}(0)>0$, for all $i \in \Gamma$ (see, e.g., Tab. I).

Theorem 1: (Protocol design rule) For any function $\hat{\chi}($. as in Assumption 1, the non trivial protocol

$$
u_{i}\left(x_{i}, x^{(i)}\right)=\frac{1}{\frac{d g\left(x_{i}\right)}{d x_{i}}} \sum_{j \in N_{i}} \phi\left(x_{j}, x_{i}\right), \quad \text { for all } i \in \Gamma
$$


lets the value $\hat{\chi}(x(t))$ be time invariant, where $\phi: \Re^{2} \rightarrow \Re$ is an antisymmetric function, i.e., $\phi\left(x_{j}, x_{i}\right)=-\phi\left(x_{i}, x_{j}\right)$.

Consider the linear function $\phi\left(x_{j}, x_{i}\right)=\alpha\left(x_{j}-x_{i}\right)$ and the different means introduced in Tab. I. The arithmetic mean is time invariant under protocol $u\left(x_{i}, x^{(i)}\right)=$ $\alpha \sum_{j \in N_{i}}\left(x_{j}-x_{i}\right)$; the geometric mean under protocol $u\left(x_{i}, x^{(i)}\right)=\alpha x_{i} \sum_{j \in N_{i}}\left(x_{j}-x_{i}\right)$; the harmonic mean under protocol $u\left(x_{i}, x^{(i)}\right)=-\alpha x_{i}^{2} \sum_{j \in N_{i}}\left(x_{j}-x_{i}\right)$; the mean of order $p$ under protocol $u\left(x_{i}, x^{(i)}\right)=\alpha \frac{x_{i}^{1-p}}{p} \sum_{j \in N_{i}}\left(x_{j}-\right.$ $\left.x_{i}\right)$.

Obviously, the time invariancy of $\hat{\chi}(x(t))$ under a given protocol does not necessarily imply that the system converges to $\hat{\chi}(x(0)) \mathbf{1}$. As it turns out at the end of the next section, for the cases in the example, the system converges to $\hat{\chi}(x(0)) \mathbf{1}$ only if $\alpha>0$ (for the harmonic mean only if $\alpha<0$ ). Dealing with means different from the arithmetic one we must also assume that $x_{i}(0)>0$ for all $i \in \Gamma$.

\section{SUFFICIENT CONDITIONS FOR CONVERGENCE}

In the previous section, we find a family of protocols as in (5) that guarantees the time invariancy of $\hat{\chi}(x(t))$. In this section, we determine sufficient conditions on the structure of functions $g($.$) and \phi($.$) such that a protocol of$ type (5) makes the system converge to $\hat{\chi}(x(0)) \mathbf{1}$ for any function $\hat{\chi}($.$) and initial state x(0)$. In particular, we prove that the system converges when the function $g($.$) is strictly$ increasing and the function $\phi($.$) is defined as follows:$

$$
\phi\left(x_{j}, x_{i}\right)=\alpha \hat{\phi}\left(\vartheta\left(x_{j}\right)-\vartheta\left(x_{i}\right)\right),
$$

where $\alpha>0$, function $\hat{\phi}: \Re \rightarrow \Re$ is continuous, locally Lipschitz, odd and strictly increasing, and function $\vartheta: \Re \rightarrow$ $\Re$ is differentiable with $\frac{d \vartheta\left(x_{i}\right)}{d x_{i}}$ locally Lipschitz and strictly positive.

Putting together (5) and (6) the resulting protocol is

$u_{i}\left(x_{i}, x^{(i)}\right)=\alpha \frac{1}{\frac{d g}{d x_{i}}} \sum_{j \in N_{i}} \hat{\phi}\left(\vartheta\left(x_{j}\right)-\vartheta\left(x_{i}\right)\right), \quad$ for all $i \in \Gamma$

Initially, we study the stability of the system under protocol (7). To this aim, we consider the space of the edge variables $\eta_{j i}=\vartheta\left(x_{j}\right)-\vartheta\left(x_{i}\right)$. It is straightforward that one point in the space of the edge variables maps infinite pairs $\left(\vartheta\left(x_{j}\right), \vartheta\left(x_{i}\right)\right)$ in the space of the state variables. We define $\eta$ as the stack vector of the edge variables, i.e, $\eta=\left\{\eta_{i j}:(i, j) \in E\right\}$. In the following lemma, we prove that $\eta=0$ is a unique equilibrium point in the space of the edge variables.

Lemma 2: (Uniqueness) Consider a network $G=(\Gamma, E)$ of dynamic agents with first-order dynamics and implement a distributed and stationary protocol $u($.$) whose components$ have the feedback form (7). Then point $\eta=0$ is a unique equilibrium point in the space of the edge variables.

We are now ready to prove that $\eta=0$ is an asymptotically stable equilibrium point, when function $g($.$) is strictly$ increasing, i.e., $\frac{d g(y)}{d y}>0$ for all $y \in \Re$.
Theorem 2: (Asymptotical Stability) Consider a network $G=(\Gamma, E)$ of dynamic agents with first-order dynamics and implement a distributed and stationary protocol whose components have the feedback form (7). If function $g($.$) is$ strictly increasing, point $\eta=0$ is an asymptotically stable equilibrium point in the space of the edge variables.

The above theorem proves that protocol (7) makes eventually the agents' trajectories align, i.e., $\lim _{t \rightarrow \infty} x_{i}(t)-$ $x_{j}(t)=0$, for any possible couples of agents $i$ and $j$. The next corollary shows that the trajectory alignment and the time invariancy of $\hat{\chi}(x(t))$ imply that trajectories converge to the desired group decision value $\hat{\chi}(x(0))$.

Corollary 1: Assume all the conditions in Theorem 2 hold. Then, $\lim _{t \rightarrow \infty} x_{i}(t)=\hat{\chi}(x(0))$ for all $i \in \Gamma$, which means that protocol (7) solves the consensus problem with respect to the group decision value $\hat{\chi}(x(0))$.

It is possible to partially relax the assumptions of Theorem 2 concerning the monotonicity of function $g($.$) . The$ reason is evident from the following theorem establishing that all agents' state trajectories are bounded.

Theorem 3: Assume all the conditions in Theorem 2 hold. Then, condition (6) implies that for all $i \in \Gamma$ and $t \geq 0$

$$
\min _{j \in \Gamma}\left\{x_{j}(0)\right\} \leq x_{i}(t) \leq \max _{j \in \Gamma}\left\{x_{j}(0)\right\} .
$$

Furthermore, any state $\tilde{x}_{i}$ within the above range is reachable under protocol (7).

Trivially, condition (8) holds even if $g(y)$ is strictly increasing only in the subset of $\Re$ defined by $\min _{j \in \Gamma}\left\{x_{j}(0)\right\} \leq y \leq \max _{j \in \Gamma}\left\{x_{j}(0)\right\}$, since the agents' state trajectory values are bounded within the same set. The boundedness of the agents' state trajectories allows us to partially relax the assumptions of Theorem 2 concerning the monotonicity of function $g($.$) . Theorem 2$ still holds if $g($.$) is strictly increasing in only a subset X \in \Re$. However, in this case, it must be true that $x_{i}(t) \in X$, for all $t \geq 0$ and for all $i \in \Gamma$. Theorem 3 proves that the latter condition is certainly satisfied if $X$ is a connected subset and $x_{i}(0) \in X$ for all $i \in \Gamma$. Theorem 2 holds even if $g($. is strictly decreasing. However, in this case, $\alpha$ in (6) must be strictly negative instead of positive.

An immediate consequence of the above considerations is the following. Since the means introduced in Tab. I have the component $g($.$) strictly increasing except the$ harmonic mean, if we consider the linear function $\phi\left(x_{j}, x_{i}\right)=\alpha\left(x_{j}-x_{i}\right)$, the system converges to $\hat{\chi}(x(0)) \mathbf{1}$ for $\alpha>0$ except for the harmonic mean where we need $\alpha<0$. Dealing with means different from the arithmetic one we also need that $x_{i}(0)>0$ for all $i \in \Gamma$, since $g(y)$ is strictly monotone for $y>0$ but not in $y=0$.

\section{Simulation Studies: Alignment Maneuver for UAVS}

We consider a team of 4 UAVs in longitudinal flight and initially at different heights. Each UAV controls the vertical rate without knowing the relative position of all UAVs but 


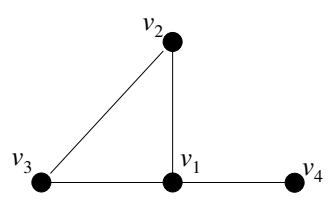

Fig. 1. The information flow in a network of 4 agents

only of neighbors according to the communication graph topology depicted in Fig. 1. For instance, the 4th UAV knows the position of only the 1 st UAV, and the 1st UAV knows the position of the 4th and 2nd UAV and so on. In the above partial information context, we are interested in determining a suitable distributed vertical rate control strategy that allows the UAVs to align their paths according to the path of a virtual leader, the formation center. In any of the four simulated alignment maneuvers, the position of the formation center is computed with respect to the positions of all UAVs respectively as the i) arithmetic mean, ii) geometric mean, iii) harmonic mean, iv) mean of order 2. The initial height is $x(0)=[5,5,10,20]^{\prime}$. We stress once again that the challenging aspect is that the UAVs know the heights of only their neighbors and are required to align their paths according to the path of the formation center, which in turns depend on the unknown position of all UAVs. In case i), (see e.g., [1], [2]) the UAVs implement the linear protocol

$$
u\left(x_{i}, x^{(i)}\right)=\sum_{j \in N_{i}}\left(x_{j}-x_{i}\right)
$$

to asymptotically align on the arithmetic mean of $x(0)$. Figure 2 shows the simulation of the longitudinal flight dynamics.

In case ii) the UAVs implement the protocol

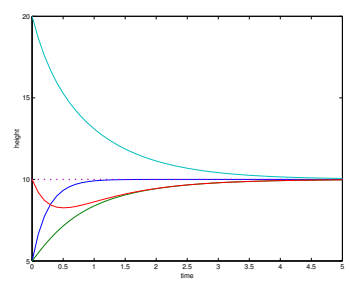

a)

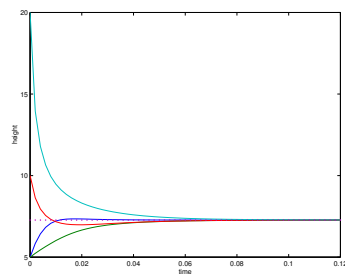

c)

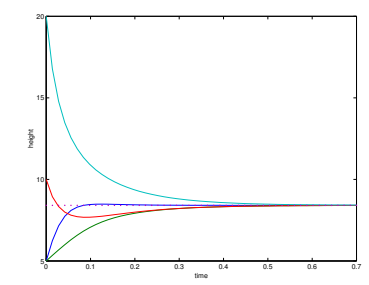

b)

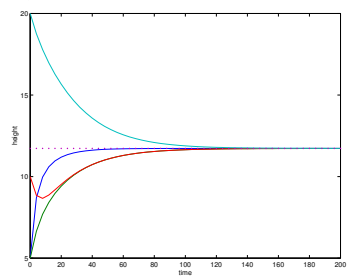

d)
Fig. 2. Longitudinal flight dynamics converging to a) the arithmetic mean under protocol (9); b) the geometric mean under protocol (10); c) the harmonic mean under protocol $(11)$; d) the mean of order 2 under protocol (12).

$$
u\left(x_{i}, x^{(i)}\right)=x_{i} \sum_{j \in N_{i}}\left(x_{j}-x_{i}\right)
$$

to asymptotically align on the geometric mean of $x(0)$. Figure 2(a)) shows the simulation of the longitudinal flight dynamics. In case iii) the UAVs implement the protocol

$$
u\left(x_{i}, x^{(i)}\right)=-x_{i}^{2} \sum_{j \in N_{i}}\left(x_{j}-x_{i}\right)
$$

to asymptotically align on the harmonic mean of $x(0)$. Figure $2(b)$ ) shows the simulation of the longitudinal flight dynamics. Finally, in case iv) the UAVs implement the protocol

$$
u\left(x_{i}, x^{(i)}\right)=\frac{1}{2 x_{i}} \sum_{j \in N_{i}}\left(x_{j}-x_{i}\right)
$$

to asymptotically align on the mean of order 2 of $x(0)$. Figure 2(c)) shows the simulation of the longitudinal flight dynamics.

Protocols (9)-(12) are characterized by different converging times (see Figs. 2). These differences are due to the fact that the protocols multiply the common term $\sum_{j \in N_{i}}\left(x_{j}-x_{i}\right)$ for different powers of $x_{i}$, respectively $1, x_{i},-x_{i}^{2}$ and $\frac{1}{2} x_{i}^{-1}$. Being $x_{i} \geq 1$ for all $i \in \Gamma$ and $t \geq 0$, the lower the power, the higher the converging time. Consider the alignment to the mean of power 2 . To obtain a converging time comparable with the one of the alignment to the arithmetic mean, we modify the protocol so that it turns to be a ratio between polynomials whose numerator is of an order greater than the denominator as in the arithmetic mean case. As an example, in Fig. 3(a)) results are reported with the protocol (12) modified as

$$
u\left(x_{i}, x^{(i)}\right)=\frac{1}{2 x_{i}} \sum_{j \in N_{i}}\left(x_{j}^{2}-x_{i}^{2}\right) .
$$

An analogous result can be obtained if we multiply the protocol (12) by twice an upper bound of $\max _{i \in \Gamma}\left\{x_{i}(0)\right\}$. The resulting scaled protocol is

$$
u\left(x_{i}, x^{(i)}\right)=\frac{\max _{i \in \Gamma}\left\{x_{i}(0)\right\}}{2 x_{i}} \sum_{j \in N_{i}}\left(x_{j}-x_{i}\right)
$$

and the corresponding longitudinal dynamics is displayed in Fig. $3(b)$ ). Observe that to implement protocol (14) the UAVs must have an a-priori knowledge or at least a bound of $\max _{i \in \Gamma}\left\{x_{i}(0)\right\}$.

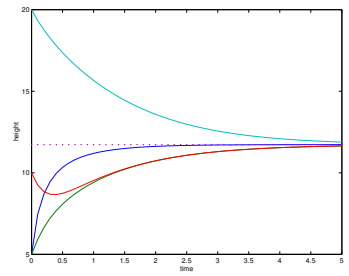

a)

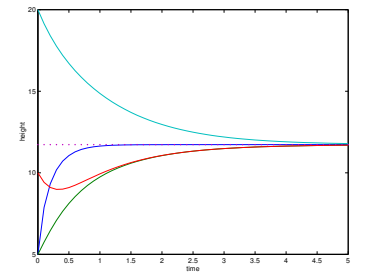

b)
Fig. 3. Longitudinal flight dynamics converging to the mean of order 2: a) under protocol (13); b) under protocol (14).

An example of alignment maneuver under protocol (14) is displayed in Fig. 4. 

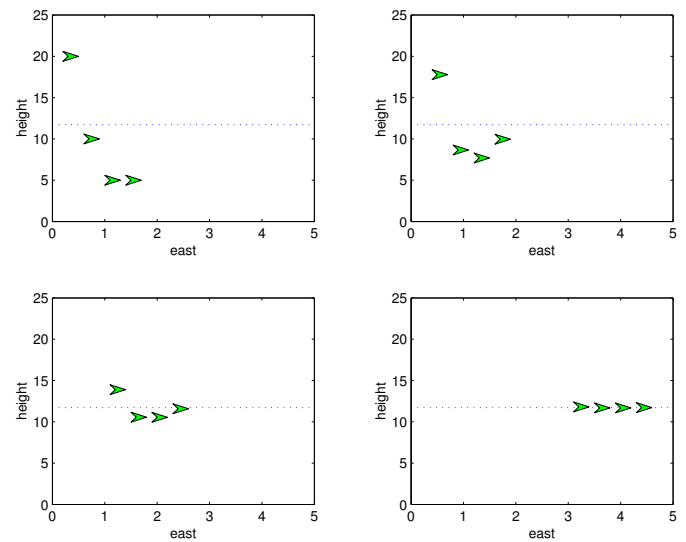

Fig. 4. Alignment to the mean of order 2 on the vertical plane.

\section{REFERENCES}

[1] R. Olfati-Saber, R. Murray, Consensus problems in networks of agents with switching topology and time-delays, IEEE Trans. on Automatic Control 49 (9) (2004) 1520-1533.

[2] D. Bauso, L. Giarrè, R. Pesenti, Attitude Alignment of a Team of UAVs under Decentralized Information Structure, in: Proc. of the IEEE Conference on Controls and Applications, Instanbul, Turkey, 2003, pp. 486-491.

[3] F. Giulietti, L. Pollini, M. Innocenti, Autonomous Formation Flight, IEEE Control Systems Magazine 20 (2000) 34-44.

[4] R. W. Beard, T. W. McLain, M. A. Goodrich, E. P. Anderson, Coordinated Target Assignment and Intercept for Unmanned Air Vehicles, IEEE Trans. on Robotics and Automation 18 (6) (2002) 911-922.

[5] H. G. Tanner, A. Jadbabaie, G. J. Pappas, Coordination of Multiple Autonomous Vehicles, in: Proc. of the 11th Mediterranean Conference on Control and Automation, also available at http://www.unm.edu/ tanner/, Rhodes, Greece, 2003.

[6] V. Gazi, K. Passino, Stability analysis of social foraging swarms, IEEE Trans. on Systems, Man, and Cybernetics 34 (1) (2004) 539557.

[7] Y. Liu, K. Passino, Stable social foraging swarms in a noisy environment, IEEE Trans. on Automatic Control 49 (1) (2004) 30-44.

[8] D. Bauso, L. Giarré, R. Pesenti, Distribuited consensus protocols for coordinated buyers, in: Proc. of the 42nd IEEE Conference on Decision and Control, Vol. 1, Maui,Hawaii, 2003, pp. 588-592.

[9] A. J. Kleywegt, V. S. Nori, M. W. P. Savelsbergh, The Stochastic Inventory Routing Problem with Direct Deliveries, Transportation Science 36 (1) (2002) 94-118.

[10] S. H. Low, F. Paganini, J. C. Doyle, Internet Congestion Control, IEEE Control System Magazine 22 (1) (2002) 28-43.

[11] A. Fax, R. M. Murray, Information flow and cooperative control of vehicle formations, in: Proc of the 15th IFAC World Congress, Barcellona,S, 2002.

[12] A. Jadbabaie, J. Lin, A. Morse, Coordination of Groups of mobile autonomous agents using nearest neighbor rules, IEEE Trans. on Automatic Control 48 (6) (2003) 988-1001.

[13] L. Moreau, Leaderless coordination via bidirectional and unidirectional time-dependent communication, in: Proc. of the 42nd IEEE Conference on Decision and Control, Maui, Hawaii, 2003, pp. 30703075 .

[14] W. Ren, R. Beard, Consensus of information under dynamically changing interaction topologies, in: Proc. of the American Control Conference, Boston, Massachussets, 2004.

[15] W. Ren, R. Beard, Consensus seeking in multi-agent systems under dynamically changing interaction topologies, in: IEEE Trans. on Automatic Control. To appear.

[16] H. G. Tanner, A. Jadbabaie, G. J. Pappas, Stable flocking of mobile agents, part ii: Dynamic topology, in: Proc. of the 42th IEEE Conference on Decision and Control, Maui, Hawaii, 2003, pp. 20162021.
[17] T. W. McLain, R. W. Beard, Coordination Variables Coordination Functions and Coooperative Timing Missions, in: Proc. of the IEEE American Control Conference, Denver, Colorado, 2003, pp. 296-301.

[18] W. Ren, R. Beard, T. McLain, Coordination variables and consensus building in multiple vehicle systems, in: Proc. of the Block Island Workshop on Cooperative Control, Springer-Verlag, 2004.

[19] R. Olfati-Saber, R. Murray, Consensus protocols for networks of dynamic agents, in: Proc. of the American Control Conference, Vol. 2, Denver, Colorado, 2003, pp. 951-956.

[20] L. Xiao, S. Boyd, Fast linear iterations for distributed averaging, Systems and Control Letters 53 (1) (2004) 65-78.

[21] D. Bauso, L. Giarrè, R. Pesenti, Nash equilibria through consensus protocols: an inventory game with threshold strategies, IEEE Trans. on Automatic Control,Submitted, available also at http://www.unipa.it/ giarre/pubblindex.

\section{APPENDIX}

Proof of Lemma 1 Assume by contradiction that $\hat{\chi}(x(t))$ is not time invariant under protocol $u($.$) and initial state$ $x(0)=a$. The consensus protocol makes the system converge to $\hat{\chi}(a)$. Let be $x\left(t_{0}\right)=b$ the state at the first time instant $t_{0}>0$ with $\hat{\chi}(a) \neq \hat{\chi}(b)$. Denote as $\bar{u}(t)$ the realization of the protocol for all $t \geq 0$. Now consider the different situation in which the protocol $u($.$) is implemented$ starting from the initial state $x(0)=b$. In this case, the consensus protocol makes the system converge to $\hat{\chi}(b)$. Denote as $\tilde{u}(t)$ the realization of the protocol for all $t \geq 0$ in this second situation. As for each agent $i$, the control depends only on $x_{i}(t)$ and $x^{(i)}(t)$ and no other information on the current or past system state, it holds $\tilde{u}(t)=\bar{u}\left(t+t_{0}\right)$, for all $t \geq 0$. Then, we obtain the following contradictory result. In the two above situations, the same controls applied starting from state $b$ make the system converge to two different group decision values, $\hat{\chi}(a)$ and $\hat{\chi}(b)$.

Proof of Theorem 1 A sufficient condition for $\hat{\chi}(x(t))$ being time invariant is that its argument $\sum_{i \in \Gamma} g\left(x_{i}(t)\right)$ is time invariant, too. The latter condition implies

$$
\sum_{i \in \Gamma} \frac{d g\left(x_{i}(t)\right)}{d t}=\sum_{i \in \Gamma} \frac{d g\left(x_{i}\right)}{d x_{i}} \dot{x}_{i}=\sum_{i \in \Gamma} \frac{d g\left(x_{i}\right)}{d x_{i}} u_{i}=0 .
$$

It is immediate to verify that protocol (5) satisfies condition $\sum_{i \in \Gamma} \frac{d g\left(x_{i}\right)}{d x_{i}} u_{i}=0$ since the antisymmetry of $\phi$ guarantees that $\sum_{i \in \Gamma} \sum_{j \in N_{i}} \phi\left(x_{j}, x_{i}\right)=0$.

Proof of lemma 2

Sufficiency. Since $\hat{\phi}($.$) is odd and strictly increasing, then$ $\hat{\phi}\left(\eta_{i j}\right)=0$ if and only if $\eta_{i j}=0$. So when $\eta=0$, the control $u_{i}$ is null for all $i \in \Gamma$. Thus, the point $\eta=0$ is an equilibrium point.

Necessity. Assume that there exists an equilibrium point $\bar{\eta} \neq 0$. We prove that such an assumption implies the existence of at least one agent $i$ with $u_{i}<0$, but this last result contradicts the definition of equilibrium for $\bar{\eta}$. Define the set of agents with maximum $\vartheta($.$) , namely,$ $I=\left\{i \in \Gamma: \vartheta\left(x_{i}\right) \geq \vartheta\left(x_{j}\right), \forall j \in \Gamma\right\}$. Trivially, $I$ is included but not equal to $\Gamma$, as $\bar{\eta} \neq 0$ implies that $i$ and $j$ with $(i, j) \in E$ such that $\vartheta\left(x_{i}\right) \neq \vartheta\left(x_{j}\right)$ exist. Since the network $G$ is connected we can always choose $i \in I$ such that there exists $j \in N_{i}$ with $\vartheta\left(x_{j}\right)<\vartheta\left(x_{i}\right)$. Now observe that, since $\hat{\phi}($.$) is an odd and strictly increasing$ 
function, then $\sum_{j \in N_{i}} \hat{\phi}\left(\vartheta\left(x_{j}\right)-\vartheta\left(x_{i}\right)\right)<0$. Actually, all the terms of the sum are non positive and at least one is strictly negative. Since it also holds that $\alpha \frac{1}{\frac{d g}{d x_{i}}} \neq 0$, the contradiction is proved.

Proof of theorem 2

We follow a line of reasoning similar to the one in [1], [19]. We prove the asymptotical stability of the equilibrium point $\eta=0$ by introducing a candidate Lyapunov function $V(\eta)=\frac{1}{2} \sum_{(i, j) \in E}\left(\psi\left(\eta_{j i}\right)+\psi\left(\eta_{i j}\right)\right)$, where $\psi: \Re \rightarrow \Re$ is $\psi(y)=\int_{0}^{y} \hat{\phi}(s) d s$. Since $\hat{\phi}$ is an odd and strictly increasing function, then $\psi(y)=0$ if and only if $y=0$, otherwise $\psi(y)>0$. As a consequence, it is immediate to verify that: a) $V(\eta)=0$ if and only if $\eta=0$; b) $V(\eta)>0$ for all $\eta \neq 0$. It remains to prove that $\dot{V}(\eta)<0$ for all $\eta \neq 0$.

$$
\begin{aligned}
\dot{V}(\eta)= & \frac{1}{2} \sum_{(i, j) \in E}\left(\hat{\phi}\left(\eta_{j i}\right) \dot{\eta}_{j i}+\hat{\phi}\left(\eta_{i j}\right) \dot{\eta}_{i j}\right)= \\
= & \frac{1}{2} \sum_{(i, j) \in E}\left(\hat{\phi}\left(\eta_{j i}\right)\left(\frac{d \vartheta\left(x_{j}\right)}{d x_{j}} \dot{x}_{j}-\frac{d \vartheta\left(x_{i}\right)}{d x_{i}} \dot{x}_{i}\right) \quad(15 \mathrm{~b})\right. \\
& \left.+\hat{\phi}\left(\eta_{i j}\right)\left(\frac{d \vartheta\left(x_{i}\right)}{d x_{i}} \dot{x}_{i}-\frac{d \vartheta\left(x_{j}\right)}{d x_{j}} \dot{x}_{j}\right)\right)=\quad(15 \mathrm{c}) \\
= & -\sum_{i \in \Gamma} \frac{d \vartheta\left(x_{i}\right)}{d x_{i}} \dot{x}_{i} \sum_{j \in N_{i}} \hat{\phi}\left(\eta_{j i}\right)= \\
= & -\sum_{i \in \Gamma} \frac{1}{\alpha} \frac{d g\left(x_{i}\right)}{d x_{i}} \frac{d \vartheta\left(x_{i}\right)}{d x_{i}} \dot{x}_{i} \frac{\alpha}{\frac{d g\left(x_{i}\right)}{d x_{i}}} \sum_{j \in N_{i}} \hat{\phi}\left(\eta_{j}(15 \mathrm{~d})\right. \\
= & -\sum_{i \in \Gamma} \frac{1}{\alpha} \frac{d g\left(x_{i}\right)}{d x_{i}} \frac{d \vartheta\left(x_{i}\right)}{d x_{i}} u_{i}^{2}
\end{aligned}
$$

In the above expression, to obtain (15c) from (15a) we simply express the derivative of the edge variables in terms of the state variables and their derivatives. To get (15d) from $(15 \mathrm{c})$, we reorder the terms and exploit the fact that $\eta_{i j}=-\eta_{j i}$ and hence $\hat{\phi}\left(\eta_{i j}\right)=-\hat{\phi}\left(\eta_{j i}\right)$. To derive (15e) from (15d) we divide and multiply each term of the external sum by $\frac{d g\left(x_{i}\right)}{d x_{i}}$, which is always different from zero by hypothesis. To obtain (15f) from (15e), we observe that both $\dot{x}_{i}$ and $\frac{\alpha}{\frac{d g\left(x_{i}\right)}{d x_{i}}} \sum_{j \in N_{i}} \hat{\phi}\left(\eta_{j i}\right)$ correspond to $u_{i}$. Finally, from (15f) we have $\dot{V}(\eta) \leq 0$ for all $\eta$, since $\frac{d g\left(x_{i}\right)}{d x_{i}}>0$ and $\frac{d \vartheta\left(x_{i}\right)}{d x_{i}}>0$ for all $x_{i}$. In particular $\dot{V}(\eta)=0$ only for $\eta=0$, since by Lemma $2 \eta=0$ if and only if $u_{i}=0$, for all $i \in \Gamma$.

Proof of Corollary 1. From Theorem 2, we can consider, without loss of generality, trajectory $\left\{x_{1}(t), t \geq 0\right\}$ of agent 1 as a limit trajectory and prove that $\lim _{t \rightarrow \infty} x_{1}\left(t_{k}\right)=$ $\hat{\chi}(x(0))$. Limit trajectory means that, for all $\varepsilon>0$ there always exists a time instant $\bar{t}(\varepsilon)$ after which all trajectories $\left\{x_{i}(t), t \geq 0\right\}$, with $i \in \Gamma, i \neq 1$ deviate from $\left\{x_{1}(t), t \geq\right.$ $0\}$ less than $\varepsilon$, i.e.,

$\forall \varepsilon>0, \exists \bar{t}(\varepsilon):\left|x_{1}(t)-x_{i}(t)\right|<\varepsilon, \forall t \geq \bar{t}(\varepsilon), \forall i \in \Gamma$.

Now, assume by contradiction that $\lim _{t \rightarrow \infty} x_{1}(t) \neq$ $\hat{\chi}(x(0))$. Since $\hat{\chi}($.$) enjoys the fixed point prop-$ erty, the previous condition is equivalent to imposing $\lim _{t \rightarrow \infty} \hat{\chi}\left(x_{1}(t) \mathbf{1}\right) \neq \hat{\chi}(x(0))$ that, in turn, implies that we can determine a value $\delta>0$ such that there exists an infinite sequence of time instants $\left\{t_{k}\right\}$, with $\lim _{k \rightarrow \infty} t_{k}=\infty$, such that $\left|\hat{\chi}\left(x_{1}\left(t_{k}\right) \mathbf{1}\right)-\hat{\chi}(x(0))\right|>\delta$. The latter condition and the continuity of function $\hat{\chi}($.$) entails the existence of an$ $\bar{\varepsilon}>0$ such that $\hat{\chi}(x) \neq \hat{\chi}(x(0))$ for all $x$ with $\mid x_{1}\left(t_{k}\right) \mathbf{1}-$ $x \mid<\bar{\varepsilon}$ for any $t_{k}$ in $\left\{t_{k}\right\}$. As we can always choose an instant $t_{s}$ in $\left\{t_{k}\right\}$ that satisfies condition (16) for $\varepsilon=\bar{\varepsilon}$, i.e, such that $t_{s} \geq \bar{t}(\bar{\varepsilon})$, we obtain that $\hat{\chi}\left(x\left(t_{s}\right)\right) \neq \hat{\chi}(x(0))$ in contradiction with the time invariancy of $\hat{\chi}(x(t))$.

Proof of Theorem 3. Consider an arbitrary value $\varepsilon>0$ and assume by contradiction that there exists a time instant $\bar{t}=\inf \left\{t \geq 0: \max _{j \in \Gamma}\left\{x_{j}(t)\right\} \geq \max _{j \in \Gamma}\left\{x_{j}(0)\right\}+\right.$ $\varepsilon)$ or $\left.\left(\min _{j \in \Gamma}\left\{x_{j}(t)\right\} \geq \min _{j \in \Gamma}\left\{x_{j}(0)\right\}-\varepsilon\right)\right\}$, namely, $\bar{t}$ is the first time instant in which (or immediately after which) either $\max _{j \in \Gamma}\left\{x_{j}(t)\right\}$ is at least $\varepsilon$ greater than $\max _{j \in \Gamma}\left\{x_{j}(0)\right\}$ or $\min _{j \in \Gamma}\left\{x_{j}(t)\right\}$ is at least $\varepsilon$ less than $\min _{j \in \Gamma}\left\{x_{j}(0)\right\}$. Here, we assume that the first of the two conditions occurs, namely, $\max _{j \in \Gamma}\left\{x_{j}(t)\right\} \geq$ $\max _{j \in \Gamma}\left\{x_{j}(0)\right\}+\varepsilon$ and we prove that $\bar{t}$ cannot exist. Symmetric argument holds if the second condition occurs. Initially, we show that $\bar{t}>0$ since $\bar{t}=0$ is contradictory with the fact that, since

$\max _{j \in \Gamma}\left\{x_{j}(0)\right\}<\infty$, all the components of $u(0)$ are bounded by definition (7). Next, we observe that all $x_{i}(t)$ are continuous and differentiable with bounded derivatives, for $0 \leq t \leq \bar{t}$, since both $\max _{j \in \Gamma}\left\{x_{j}(t)\right\}$ and $\min _{j \in \Gamma}\left\{x_{j}(t)\right\}$ are bounded and then all the components $u(t)$ are bounded too. Consequently, $y(t)=$ $\max _{j \in \Gamma}\left\{x_{j}(t)\right\}$ is a continuous function, for $0 \leq t \leq \bar{t}$, and cannot present a discontinuity between $\bar{t}$ and $\bar{t}^{+}$, then $y(\bar{t})=\max _{j \in \Gamma}\left\{x_{j}(0)\right\}+\varepsilon$.

Now define as switching instants $0, \bar{t}$ and all the instants $t_{k}, 0 \leq t_{k} \leq \bar{t}$, such that

$\arg \max _{j \in \Gamma}\left\{x_{j}\left(t_{k}\right)\right\} \neq \arg \max _{j \in \Gamma}\left\{x_{j}\left(t_{k}^{+}\right)\right\}$, where the latter function always returns the minimum index that satisfies the maximality condition. In other worlds, $t_{k}$ are time instants in which a trajectory $x_{i}(t)$ loses its maximality. Consider the generic $i$ such that $i=\max _{j \in \Gamma}\left\{x_{j}(t)\right\}$ between two consecutive switching instants $t_{k}, t_{k+1}$. In this case, $u_{i}(t) \leq 0$ by definition (7) for all $t_{k} \leq t \leq t_{k+1}$. Hence the continuity and differentiability of $x_{i}(t)$ implies $y\left(t_{k}\right)=x_{i}\left(t_{k}\right) \geq x_{i}\left(t_{k+1}\right)=y\left(t_{k+1}\right)$. In this context, define $\delta_{k}=x_{i}\left(t_{k+1}\right)-x_{i}\left(t_{k}\right)$. Finally, observe that, as $y(t)$ is continuous, we can write $y(\bar{t})=y(0)+\sum_{k: t_{k} \in T} \delta_{k}$, where $T$ is the set of the switching instant different from $\bar{t}$. As all $\delta_{k} \leq 0$, we obtain the contradictory result that $y(0)+\varepsilon \leq y(\bar{t}) \leq y(0)$. Hence, $y(\bar{t})$ and $\bar{t}$ cannot exist. To complete the prove note that the above arguments hold for any value of $\varepsilon>0$. It is left to show that any state $\tilde{x}_{i}$ within the above range is reachable under protocol (7). But this is easy to see since $\tilde{x}_{i}$ can be expressed as convex combination of $x_{j}(0)$ for all $j \in \Gamma$. Hence, it is sufficient to choose $\chi=\tilde{x}_{i}=\sum_{j \in \Gamma} \alpha_{j} x_{j}(0)$ with $0 \leq \alpha_{j} \leq 1$ for all $j \in \Gamma$. 